\title{
Influence of active nitrogen species on surface and optical properties of epitaxial GaN films
}

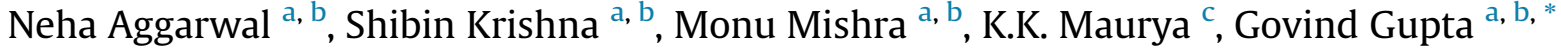 \\ a Physics of Energy Harvesting Division, CSIR-National Physical Laboratory, Dr. K.S. Krishnan Marg, New Delhi, 110012, India \\ ${ }^{\mathrm{b}}$ Academy of Scientific and Innovative Research (AcSIR), CSIR-NPL Campus, Dr. K.S. Krishnan Marg, New Delhi, 110012, India \\ c Sophisticated and Analytical Instrumentation, CSIR-National Physical Laboratory, Dr. K.S. Krishnan Marg, New Delhi, 110012, India
}

\section{A R T I C L E I N F O}

Article history:

Received 9 October 2015

Received in revised form 16 November 2015

Accepted 28 November 2015

Available online 2 December 2015

\section{Keywords:}

GaN

Homoepitaxy

Plasma power

PAMBE

\begin{abstract}
A B S T R A C T
Influence of active nitrogen species on surface and optical properties of homoepitaxial GaN films grown on GaN epilayers has been investigated. The epitaxial GaN films were grown at varying plasma powers $(350-500 \mathrm{~W})$ under identical growth conditions. High resolution X-Ray diffraction, Field Emission Scanning Electron Microscopy, Atomic Force Microscopy and Photoluminescence measurements were employed to characterize the structural, morphological and optical properties of the grown GaN films. High plasma power $(500 \mathrm{~W}$ ) lead to an increment in active nitrogen radicals and yielded high crystalline quality with reduced dislocations compared to low plasma power $(350,400 \mathrm{~W}$ ) which divulge the presence of metallic gallium on the surface and low roughness. The valence band maximum position, electron affinity and ionization energy of the films increased with increment in plasma power. PL measurements revealed narrow and intense band to band edge emission with negligible defect related yellow band peak for the sample grown at $500 \mathrm{~W}$. The analysis conveyed that higher amount of active nitrogen species encouraged good optical properties with insignificant defect states which can be employed for the fabrication of high performance optoelectronic \& photovoltaic devices.
\end{abstract}

() 2015 Elsevier B.V. All rights reserved.

\section{Introduction}

III-Nitride semiconducting materials have gained huge attention in the field of optoelectronics and photonics [1-3] due to their noteworthy properties like wide direct band gap, high thermal conductivity and good thermal stability [4]. Plasma-Assisted Molecular Beam Epitaxy (PAMBE) technique has been employed for the growth of III-Nitride material system for subsequent design and development of efficient devices from past few decades. PAMBE growth offers very high crystalline quality, deposition at lower temperature, sharp interfaces and smooth surfaces with a precise control over the growth parameters [4-8]. Radio frequency (rf) inductively coupled plasma source has extensively been used as nitrogen activator source for the production of active nitrogen species $[4,6]$. The rf-plasma sources are advantageous as they create higher amount of favourable active nitrogen species $\left(\mathrm{N}\right.$ or $\mathrm{N}^{*}$ ) compared to the molecular nitrogen $\left(\mathrm{N}_{2}\right.$ or $\left.\mathrm{N}_{2}^{+}\right)$[5]. It was reported

\footnotetext{
* Corresponding author: Physics of Energy Harvesting Division, CSIR-National Physical Laboratory, Dr. K.S. Krishnan Marg, New Delhi, 110012, India.

E-mail address: govind@nplindia.org (G. Gupta).
}

that the rf-plasma sources enables high growth rate, low ion damage, high temperature operation and superior optical properties [9-11]. Jmerik et al. reported that the intensity of the flux of activated nitrogen originated from rf-plasma source can be linearly controlled, and an increment in the rf-plasma power lead to a higher amount of active nitrogen species with high growth rate [12]. Various studies have been performed to understand and analyse the role of nitrogen species on the growth of GaN films [5,12-14]. McSkimming et al. demonstrated that the growth rates of GaN films grown by PAMBE can be tailored and a growth rate of $2.6 \mu \mathrm{m} / \mathrm{h}$ can be achieved by varying the plasma conditions [15]. Tarsa et al. reported that the structure and morphology of the GaN films grown via PAMBE is extremely sensitive to the III/V ratio by varying the nitrogen flux [16]. The surface structure can be varied from Ga-stable regime (flat) to an intermediate or N-stable (island) growth regime. A strong dependence and direct correlation between deep level defect densities with plasma induced atomic and ionic nitrogen species was also reported [17].

In the present study, we report the influence of rf-plasma power generated active nitrogen species on the structural and optical properties of homoepitaxial GaN films. Surface properties were 
scrutinized via microscopic measurements (FESEM, AFM). Structural defects, surface morphology, and optical properties of the grown films were thoroughly analysed and correlated. The electronic structure and oxygen chemisorption is probed via X-Ray and Ultraviolet photoemission spectroscopy (XPS and UPS).

\section{Experimental}

Ultrahigh vacuum (UHV) based Riber compact 21 PAMBE system, operating at base pressure in the range of $2 \mathrm{E}-11$ Torr was used for the growth of epitaxial GaN films. An rf-plasma source (Addon) was attached to provide the active nitrogen species during the growth. Gallium (Ga) flux was supplied via heating the standard Knudsen cells to $980{ }^{\circ} \mathrm{C}$, which resulted in a beam equivalent pressure (BEP) of 1.2 E-6 Torr. The homoepitaxial growth of GaN films were performed on commercially purchased MOCVD grown $3.5 \mu \mathrm{m}$ thick GaN epilayers on c-plane sapphire substrate (MGcS). The ex-situ wet chemical cleaning of the substrates was performed via standard cleaning procedure [18]. The GaN films (thickness varied from 0.8 to $1.1 \mu \mathrm{m}$ ) were grown at a constant substrate temperature of $735{ }^{\circ} \mathrm{C}$ with plasma power varying from $350 \mathrm{~W}$ to $500 \mathrm{~W}$, keeping all other parameters as constant. The samples are abbreviated as S1, S2 and S3 for films grown at 350, 400 and $500 \mathrm{~W}$ respectively.

Various characterization tools have been employed ex-situ to analyse the quality of the homoepitaxially grown GaN films. The crystalline quality has been ascertained by high-resolution x-ray diffraction (HRXRD, Pananalytical, X'Pert PRO MRD System) while the morphological and topographical properties were examined by using field-emission scanning electron microscopy (FESEM, ZEISS AURIGA) and atomic force microscopy (AFM, V-Veeco). XPS and UPS (Omicron Multiprobe Surface Analysis System) measurements were carried out to scrutinize the surface chemistry, energy band structure and Fermi level (FL) position of the grown films. Monochromatized AlK $\alpha(1486.7 \mathrm{eV})$ and $\mathrm{He}(\mathrm{I})(21.2 \mathrm{eV})$ radiation source was employed for XPS and UPS measurements, respectively. Using He-Cd laser as an excitation source of $325 \mathrm{~nm}$, the room temperature (RT) photoluminescence (PL) has been performed on the samples to examine the optical properties and presence of defect states in the grown samples.

\section{Results and discussion}

Fig. 1 (a) shows the 2 theta-omega scan of the grown GaN film taken along (0002) plane representing the first and second order diffraction peaks of GaN and sapphire. The sharp peaks revealed the high crystalline quality of the grown epitaxial GaN film. Fig. 1 (b) represents a couple of curves plotted between the plasma power and the full width at half maximum (FWHM) of X-Ray rocking curves (RC) taken along (002) and (102) plane of diffraction given by $(\boldsymbol{\Delta})$ and $(\star)$ symbol in the plot which will give a measure of screw and edge dislocations respectively. Since, the screw dislocations are developed by following a loop of atoms around dislocation line resulting into one plane up or down in the lattice and can be propagated from the template to the MBE grown GaN films. Thus, the variation in FWHM is almost negligible in RC taken along (002) plane from S1 to S3. However, the FWHM value of peak in RC taken along (102) plane was significantly reduced from Ga - stable to intermediate regime as the edge dislocations were formed due to growth of an extra half plane of atoms into the crystal lattice and cannot propagate into the overgrown film. Tsai et al. also reported that edge dislocations are dominant in Ga-stable growth regime [19]. The obtained screw and edge dislocation densities were further integrated to evaluate the total threading dislocation density (TDD) in the grown GaN films [18]. Hence, via using the FWHM values measured from RC of grown samples, the TDD was calculated to be $2.65 \times 10^{7} \mathrm{~cm}^{-2}, 2.62 \times 10^{7} \mathrm{~cm}^{-2}$ and $2.4 \times 10^{7} \mathrm{~cm}^{-2}$ for S1, S2 and S3 respectively. It was observed that the TDD for S3 was least when compared to other GaN films. Further, to evaluate the effect of reduced TDD at varied plasma power on the topographical properties of the grown film, the surface of the films has been probed by using FESEM and AFM.

Recent report suggested that the surface structure of the GaN films are highly affected by the $\mathrm{Ga} / \mathrm{N}$ flux (i.e. plasma power at constant Ga flux) [14]. Fig. 2 (a-c) represent the surface morphology of the grown GaN films (S1 to S3) examined by FESEM displaying the step-flow growth. S1 shows large amount of small hexagonal pits (having an average pit size of $\sim 50 \mathrm{~nm}$ ) with metallic Ga droplets on its surface. The hexagonal pits are formed due to the existence of screw dislocations and indicate that the films grown on template are epitaxial wurtzite GaN (0001) films [20]. In the case of S2, the amount of metallic Ga droplets has been reduced which was attributed to the consumption of excess metallic Ga by the increased amount of active nitrogen species to form GaN with reduced pit density. However, the average size of the pit was observed to be doubled $(\sim 100 \mathrm{~nm})$. On further increasing the plasma power to $500 \mathrm{~W}$, there were no traces of metallic Ga droplets, though a few bigger hexagonal pits (shown in the rightbottom inset of Fig. 2 (c)) possessing an average size of $\sim 200 \mathrm{~nm}$ were observed. The increase in average pit size infers that stress relaxation might occur during the transition from $\mathrm{Ga}$ - stable to intermediate regime with increasing plasma power. The surface topography of the GaN films were also scrutinized using AFM measurements. The $1 \times 1 \mu \mathrm{m}^{2}$ AFM images (Inset of Fig. $2(\mathrm{a}-\mathrm{c})$ )

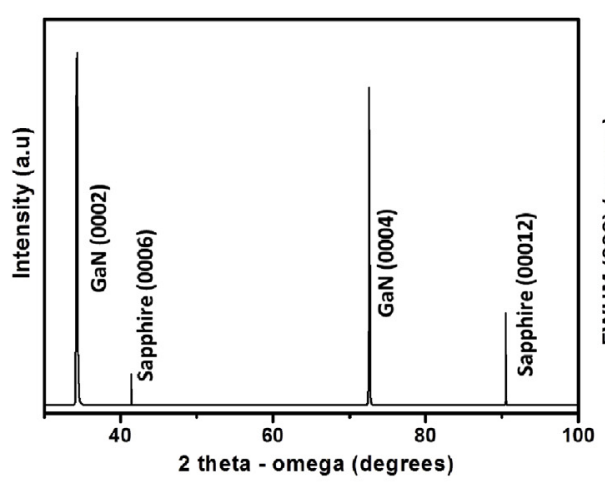

(a)

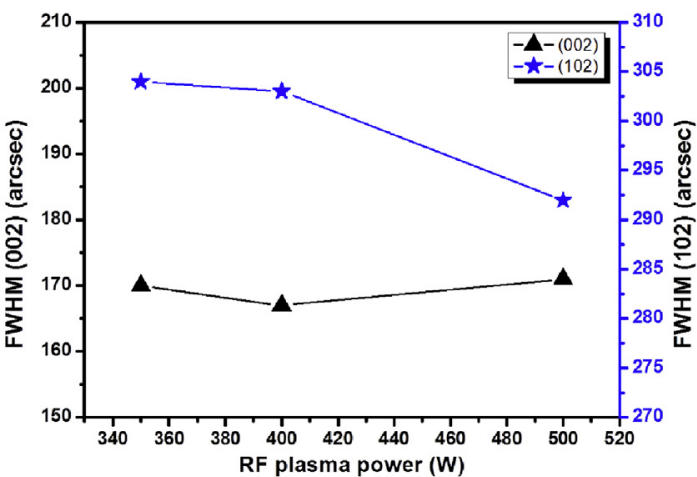

(b)

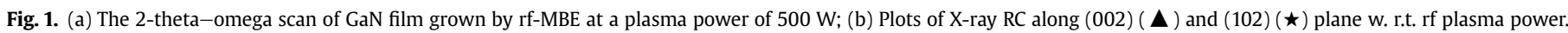



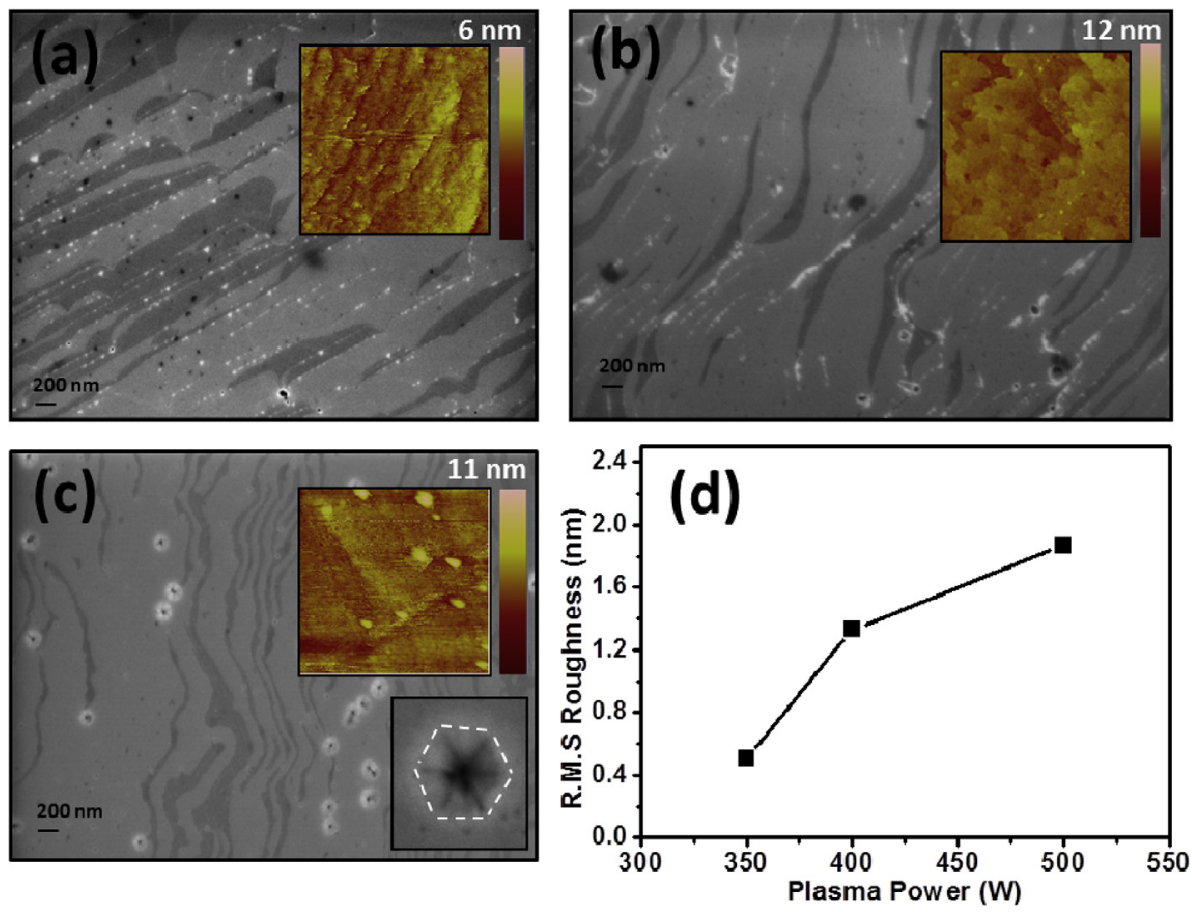

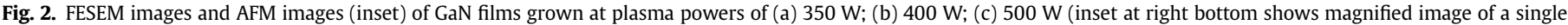
hexagonal pit) and (d) shows a curve representing variation in r.m.s. roughness with plasma power.

represents that the step flow growth was adopted which was in accordance with FESEM results discussed above. It was reported that the surface structure is closely related to the dislocations present in the films [21]. HRXRD results concluded that S3 possess least amount of TDD, which is well supported via the obtained surface structures ascertained by AFM measurements.

Since all the samples were grown on identical substrate temperature (i.e. $735^{\circ} \mathrm{C}$ ) at constant Ga flux, the change in surface morphology was attributed to variation in plasma power. Recent study reported that metallic gallium present on the surface assists in smoothening of surface along with covering the pits [22]. Thus, the increment in r.m.s. roughness was governed by the reduction in excess Ga from the surface of the film. The presence of excess Ga on the surface provides a wetting layer and smoothen the surface of the GaN film. The density functional calculation performed via Zywietz et al. demonstrated that the metallic gallium on surface possess very low migration barrier and high adatom diffusivity [23]. Thus, surface having metallic gallium may support the migration of adatoms and lead to a smoother morphology with low r.m.s. roughness. The AFM measurements divulged step flow growth in the samples which is well supported via FESEM results. Fig. 2 (d) shows a plot representing the variation in r.m.s. roughness with plasma power. The profile represents that r.m.s. roughness increases from 0.51 to $1.87 \mathrm{~nm}$ as the plasma power increased from $350 \mathrm{~W}$ to $500 \mathrm{~W}$. The values of r.m.s. roughness, average pit density and average pit size are tabulated in Table 1 for GaN films grown at different plasma powers.
Furthermore, photoemission analysis has been carried out to study the influence of active nitrogen species on the surface chemistry and electronic properties of the films. Fig. 3(a) shows the $\mathrm{Ga}-\mathrm{O} / \mathrm{Ga}-\mathrm{N}$ ratio of the grown films calculated from convoluted XPS Ga (3d) core level spectra (not shown here). The Ga-O/Ga-N ratio was obtained to be $0.17,0.26$ and 0.41 for S1, S2 and S3 respectively. Fig. 3(b) shows the analysis of energy band structure, obtained via UPS measurements of the films. The VB maximum (VBM) (i.e. separation between FL and the top of valence band) position was determined by extrapolating a linear fit to the leading edge of the valence band photoemission to the baseline [24-26]. The VBM was calculated to be $3.9 \pm 0.1 \mathrm{eV}, 4.0 \pm 0.1 \mathrm{eV}$ and $4.2 \pm 0.1 \mathrm{eV}$ for S1, S2 and S3 respectively. The UPS measurements divulged higher value of VBM (above conduction band minimum) which corresponds to the higher amount of surface oxide $[27,28]$. The electron affinities $(\chi)$ of the grown films were calculated from the UPS spectra via following equation:

$\chi=\mathrm{h} v-\mathrm{W}-\mathrm{E}_{\mathrm{g}}$

where, $\mathrm{h} v$ is incident photon energy, $\mathrm{W}$ is spectral width and $\mathrm{E}_{\mathrm{g}}$ is the band gap $(3.4 \mathrm{eV})$ of the material. The aforementioned calculation yielded electron affinities of 5.2, 5.3 and $5.5 \mathrm{eV}$ for S1, S2 and $\mathrm{S} 3$ respectively. The ionization energy (sum of electron affinity and band gap) were observed to be $8.6,8.7$ and $8.9 \mathrm{eV}$ for 350,400 and $500 \mathrm{~W}$ grown films. The high value of electron affinity and ionization energy indicates higher amount of surface oxide $[22,27,28]$.

Table 1

Values of r.m.s. Roughness, Avg. Pit Density and Avg. Pit size of samples grown at different plasma powers by rf-MBE.

\begin{tabular}{|c|c|c|c|c|}
\hline S.No. & Rf-power (W) & r.m.s Roughness (nm) & Avg. pit density $\left(\mathrm{x} 10^{8} \mathrm{~cm}^{-2}\right)$ & Avg. pit size (nm) \\
\hline 1. & 350 & 0.51 & 2.4 & 50 \\
\hline 2. & 400 & 1.33 & 1.6 & 100 \\
\hline 3. & 500 & 1.87 & 1.0 & 200 \\
\hline
\end{tabular}



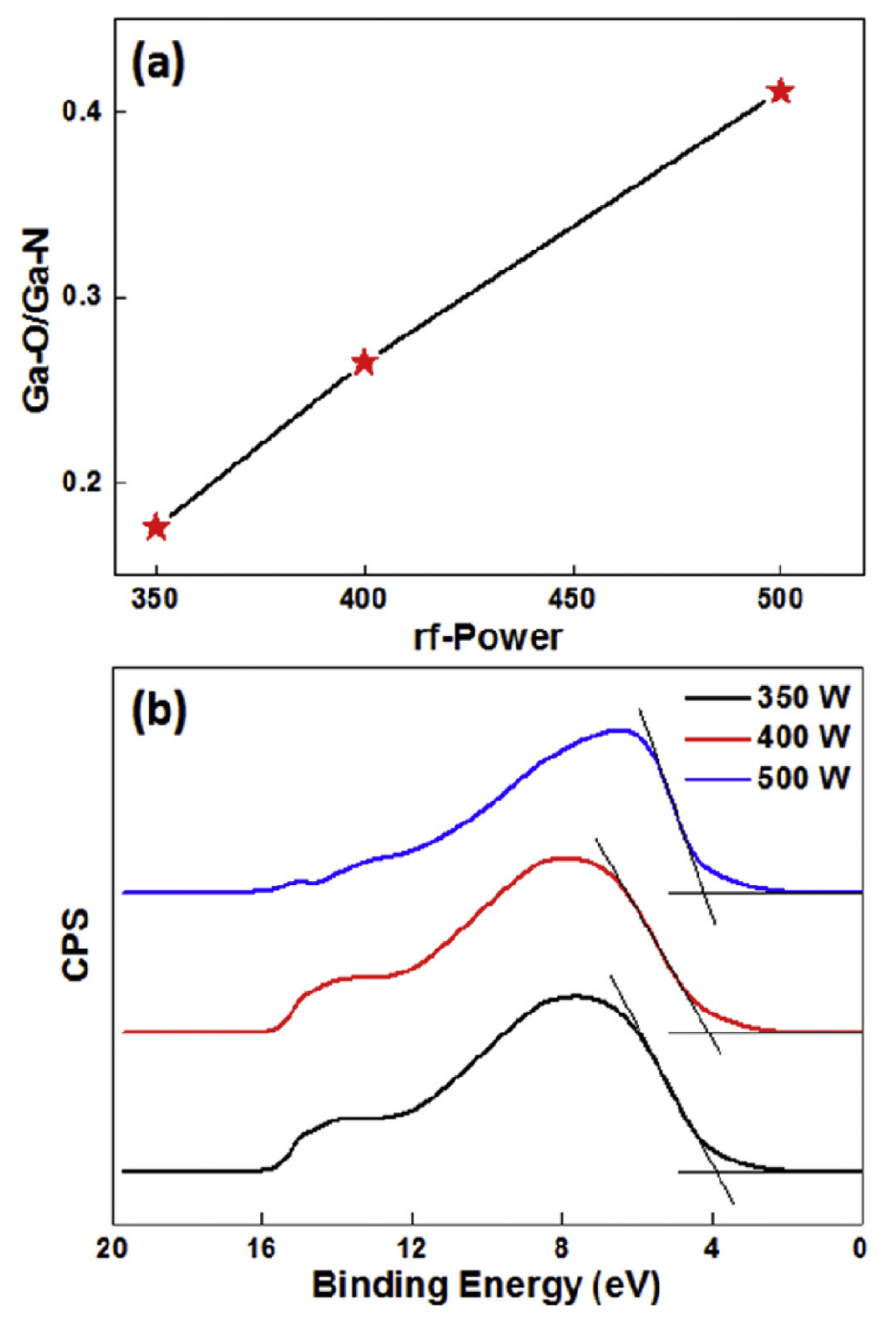

Fig. 3. (a) Ga-O/Ga-N ratio and (b) UPS valence band spectra of the films grown at different plasma powers.

Thus, photoemission measurements confirmed that the contribution of surface oxide was increased with increase in plasma power. Further, the adsorption of oxygen on the surface of the films can be directly correlated with increment in surface roughness. The presence of metallic gallium on the surface of the films grown on low plasma power increases the adatom mobility and favours migration of atoms in order to reduce the surface free energy [23]. However, higher surface oxide present in the films leads to reduction in surface migration and increases the surface roughness [22]. This is the possible reason for enhanced surface roughness observed in the films grown at higher plasma power as evident from the AFM analysis.

The optical properties of the homoepitaxial GaN films were analysed via RT-PL spectra as shown in Fig. 4. A sharp and highly intense near band edge (NBE) emission peak was perceived at $361.76 \mathrm{~nm}$ for S3. A comparatively low intense and broader NBE peaks was observed for S1 and S2. The NBE peak position for S2 was same as S3, however for S1 the NBE position is found to be at $361.65 \mathrm{~nm}$. Generally, the PL band - edge peak position will shift towards higher wavelength (in comparison to the unstressed bulk GaN film at $\sim 364.4 \mathrm{~nm}$ ) as the compressive stress relaxation occurs in GaN film [29]. Thus, the shift witnessed in PL peak position of grown GaN samples with increasing plasma power infers relaxation of stress in the MBE grown films. This relaxation in the film endorses the increase in pits size as shown in Fig. 2(a-c). The PL

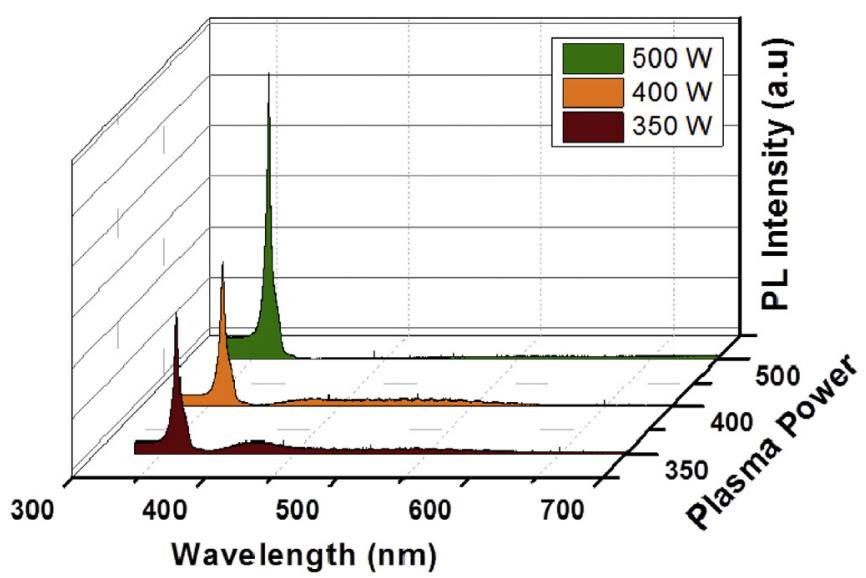

Fig. 4. RT-Photoluminescence spectra of GaN films grown at different plasma powers.

line widths were calculated to be $38.7 \mathrm{meV}$ and $37.6 \mathrm{meV}$ for S1 and $\mathrm{S} 2$, while it is reduced to $34 \mathrm{meV}$ for S3. The reduction in PL line width defines an increase in crystalline nature of the film, which is in agreement with the reduced dislocation density (TDD) as affirmed by HRXRD measurements. S3 displayed high crystalline quality, low TDD and a sharp and intense PL band to band edge emission. This implies that a highly crystalline GaN film with good optical properties has been grown at a plasma power of $500 \mathrm{~W}$. A defect related Yellow band (YB) emission (near $\sim 550 \mathrm{~nm}$ ) was also observed in S1 and S2. The YB emission is a consequence of several gallium vacancy related defects in undoped GaN [30]. However, the YB emission was diminished for the GaN films grown at $500 \mathrm{~W}$, attributing to low gallium vacancy related defects. The analysis demonstrated that high plasma power (i.e. $500 \mathrm{~W}$ ) lead to high quality GaN films with sharp NBE and low defects.

In summary, we observed that an increase in plasma power lead to high active nitrogen species and enhances the film quality. The films grown at low plasma power exhibit some metallic gallium species which gets incorporated by forming $\mathrm{GaN}$ on further increasing the plasma power and thus, increases the r.m.s. roughness. The chemisorption of oxygen was increased significantly for the samples grown at high plasma power and yielded a higher surface roughness. The films grown on high plasma power also displays a sharp NBE with low YB related defects.

\section{Conclusion}

A dominant contribution of active nitrogen species during the homoepitaxial growth of GaN films was asserted. It is observed that the increment in plasma power lead to the growth of high quality GaN films with low TDD. Films grown at low plasma power exhibited metallic gallium on the surface with low r.m.s. roughness. However, at high plasma power, the metallic gallium bond with active $\mathrm{N}$ species to form GaN on the surface of the film. An increment in the plasma power lead to higher amount of surface oxide and increased electron affinities. The highly intense and sharp main peak of PL along with the absence of defect bands revealed good quality GaN film grown at rf-plasma power of $500 \mathrm{~W}$.

\section{Acknowledgement}

The authors are thankful to Director, CSIR-NPL India for his constant encouragement and immense support. This work is financially supported by Council of Scientific and Industrial Research, India under the XIIth-FYP-PSC-0109. The authors would 
like to thank Mrs. Mandeep Kaur and Dr. Ved Varun Aggarwal for FESEM and AFM measurements, respectively. One of the co-author (SK) would like to thank DST (GoI) and SIMCO Global Tech. \& System Ltd. for financial support under Prime Minister Doctoral Fellowship.

\section{References}

[1] S. Nakamura, M. Senoh, N. Iwasa, S. Nagahama, High-brightness InGaN blue, green and yellow light-emitting diodes with quantum well structures, Jpn. J.Appl. Phys. 34 (1995) L797.

[2] T. Doi, Current status and future prospects of GaN substrates for green devices, Sens. Mater. 25 (2013) 141

[3] A.K. Pantazis, G. Konstantinidis, E. Gizeli, Characterization of a GaN lamb-wave sensor for liquid-based mass sensing applications, IEEE Sens. J. 14 (2014) 908

[4] X. Wang, A. Yoshikawa, Molecular beam epitaxy growth of GaN AlN and InN, Prog. Cryst. Growth Charact. Mater. 48 (2004) 42.

[5] T. Kikuchi, A.S. Somintac, O. Ariyada, M. Wadaa, T. Ohachi, Role of excited nitrogen species in the growth of GaN by RF-MBE, J. Cryst. Grow. 292 (2006) 221.

[6] K. Klosek, M. Sobanska, G. Tchutchulashvilli, Z.R. Zytkiewicz, H. Teisseyre, L. Klopotowski, Optimization of nitrogen plasma source parameters by measurements of emitted light intensity for growth of GaN by molecular beam epitaxy, Thin Sol. Films 534 (2013) 107.

[7] T.C. Shibin Krishna, N. Aggarwal, G.A. Reddy, P. Dugar, M. Mishra, L. Goswami, N. Dilawar, M. Kumar, K.K. Maurya, G. Gupta, Probing the correlation between structure, carrier dynamics and defect states of epitaxial GaN film on (11-20) sapphire grown by rf-molecular beam epitaxy, RSC Adv. 5 (2015) 73261.

[8] T.C. Shibin Krishna, N. Aggarwal, M. Mishra, K.K. Maurya, M. Kaur, G. Seghal, S. Singh, N. Dilawar, B.K. Gupta, G. Gupta, Eptaxial growth of high In-content $\operatorname{In}_{0.41} \mathrm{Ga}_{0.59} \mathrm{~N} / \mathrm{GaN}$ heterostructure on (11-20) Al2O3 substrate, J. Alloy Compd. 658 (2016) 470

[9] W.C. Hughes, W.H. Rowland Jr., M.A.L. Johnson, S. Futija, J.W. Cook Jr. J.F. Schetzina, J. Ren, J.A. Edmond, Molecular bean epitaxy growth and properties of GaN films on GaN/SiC substrates, J. Vac. Sci. Tech. B 13 (1995) 1571

[10] V. Kirchner, H. Heinke, U. Birkle, S. Einfeldt, D. Hommel, H. Selke, P.L. Ryder, Ion induced crystal damage during plasma assisted MBE growth of GaN layers, Phys. Rev. B 58 (1998) 15749.

[11] A.V. Blant, O.H. Hughes, T.S. Cheng, S.V. Novikov, C.T. Foxon, Nitrogen species from radio frequency plasma sources used for molecular beam epitaxy growth of GaN, Plasma Sources Sci. Tech. 9 (2000) 12.

[12] V.N. Jmerik, A.M. Mizerov, T.V. Shubina, S.B. Listoshin, S.V. Ivanov, Controlling active nitrogen flux in plasma-assisted molecular beam epitaxy of group III nitrides, Tech. Phys.Lett. 33 (2007) 333.

[13] J. Osaka, M. Senthil Kumar, H. Toyoda, T. Ishijima, H. Sugai, T. Mizutani, Role of atomic nitrogen during GaN growth by plasma-assisted molecular beam epitaxy revealed by appearance mass spectrometry, Appl. Phys. Lett. 90 (2007) 172114.
[14] Y. Kawai, S. Chen, Y. Honda, M. Yamaguchi, H. Amano, H. Kondo, M. Hiramatsu, H. Kano, K. Yamakawa, S. Den, M. Hori, Achieving high-growthrate in GaN homo-epitaxy using high-density nitrogen radical source, Phys. Sta. Sol. C 8 (2011) 2089.

[15] B.M. McSkimming, F. Wu, T. Huault, C. Chaix, J.S. Speck, Plasma assisted molecular beam epitaxy of $\mathrm{GaN}$ with growth rates $>2.6 \mathrm{~mm} / \mathrm{h}$, J. Crys. Grow. 386 (2014) 168.

[16] E.J. Tarsa, B. Heying, X.H. Wu, P. Fini, S.P. DenBaars, J.S. Speck, Homoepitaxial growth of GaN under Ga-stable and N-stable conditions by plasma-assisted molecular beam epitaxy, J. Appl. Phys. 11 (1997) 82.

[17] A.R. Arehart, C. Poblenz, J.S. Speck, S.A. Ringel, Effect of nitrogen plasma power on defects levels in Ni/n-GaN Schottky diodes grown by molecular beam epitaxy, J. Appl. Phys. 107 (2010) 054518.

[18] N. Aggarwal, T.C. Shibin Krishna, L. Goswami, M. Mishra, G. Gupta, K.K. Maurya, S. Singh, N. Dilawar, M. Kaur, Extenuation of stress and defects in GaN films grown on a metal - organic chemical vapor deposition-GaN/csapphire substrate by plasma-assisted molecular beam epitaxy, Crys. Grow. Des. 15 (2015) 2144.

[19] J. Tsai, I. Lo, K. Chuang, L. Tu, J. Huang, C. Hsieh, K. Hsieh, Effect of N to Ga flux ratio on the GaN surface morphologies grown at high temperature by plasma assisted molecular beam epitaxy, J. Appl. Phys. 95 (2004) 460.

[20] J. Kim, K.H. Bai, Characterization of hexagonal defects in gallium nitride on sapphire, J. Ceram. Pro. Res. 8 (2007) 277.

[21] B. Heying, E.J. Tarsa, C.R. Elsass, P. Fini, S.P. Denbaars, J.S. Speck, Dislocation mediated surface morphology of GaN, J. Appl. Phys. 85 (1999) 6470.

[22] M. Mishra, T.C. Shibin Krishna, N. Aggarwal, M. Kaur, S. Singh, G. Gupta, Pit assisted oxygen chemisorption on GaN Surfaces, Phys. Chem. Chem. Phys. 17 (2015) 15201.

[23] T. Zywietz, J. Neugebauer, M. Scheffler, Adatom diffusion at GaN (0001) and (0001) surfaces, Appl. Phys. Lett. 73 (1998) 487.

[24] M. Mishra, T.C. Shibin Krishna, P. Rastogi, N. Aggarwal, A.K.S. Chauhan, L. Goswami, G. Gupta, New approach to clean GaN Surfaces, Mater. Focus 3 (2014) 218.

[25] M. Mishra, T.C. Shibin Krishna, N. Aggarwal, S. Vihari, A.K.S. Chauhan, G. Gupta, A comparative photoelectron spectroscopic analysis of MBE and MOCVD grown epitaxial GaN films, Sci. Adv. Mater. 7 (2015) 546.

[26] M. Mishra, T.C. Shibin Krishna, M. Kumar, G. Gupta, Origin of surface electron accumulation and fermi level pinning in low energy ion induced InN/GaN heterostructure, Mater. Chem. Phys. 162 (2015) 640.

[27] M. Mishra, T.C. Shibin Krishna, N. Aggarwal, G. Gupta, Surface chemistry and electronic structure of nonpolar and polar GaN films, Appl. Surf. Sci. 345 (2015) 440.

[28] M. Mishra, T.C. Shibin Krishna, N. Aggarwal, S. Vihari, G. Gupta, Electronic structure analysis of GaN films grown on $\mathrm{r}-$ and a-plane sapphire, J. Alloy Compd. 645 (2015) 230.

[29] W. Rieger, T. Metzger, H. Angerer, R. Dimitrov, O. Ambacher, M. Stutzmann, Appl. Phys. Lett. 68 (1996) 970.

[30] J. Neugebauer, C.G.V. Walle, Gallium vacancies and the yellow luminescence in GaN, Appl. Phys. Lett. 69 (1996) 4. 